\title{
Compartmental Surgery for Oral Tongue Cancer: Objective and Subjective Functional Evaluation
}

\author{
Alberto Grammatica, MD (1); Cesare Piazza, MD (1); Nausica Montalto, MD; Francesca Del Bon, MD; \\ Barbara Frittoli, MD; Monica Mazza, MD; Alberto Paderno, MD (D); Davide Lancini, MD; Milena Fior, MD; \\ Alberto Deganello, MD, PhD (D); Davide Lombardi, MD (D); Piero Nicolai, MD
}

\begin{abstract}
Objective: To assess functional outcomes in patients treated by compartmental tongue surgery (CTS) and reconstruction for advanced oral tongue/floor-of-mouth cancer.

Study Design: Retrospective case series.

Methods: A retrospective cohort of patients $(n=48)$ treated by CTS and free flap reconstruction was prospectively evaluated concerning postoperative functional outcomes at different time points ( 6 months and 1 year). Swallowing was studied by videonasal endoscopic evaluation (VEES) and videofluoroscopy (VFS), testing various food consistencies and grading the results with the Donzelli scale. Speech articulation, lingual strength, and endurance were studied by phone call and Iowa Oral Performance Instrument (IOPI). Subjective tests (EORTC H\&N35 and UWQOL) were administered.

Results: After 1 year, VEES showed a Donzelli scale of $67 \%$ level 1, 23\% level 2, and 10\% level 3. Vallecular pouch was present in $81 \%$ of patients. VFS showed levels 1, 2, and 3 in $42 \%, 25 \%$, and 33\%, respectively, with liquids (L); $48 \%, 19 \%$, and $33 \%$, with semi-liquids (SL); and 54\%, 33\%, and 13\%, with semi-solids (SS). Vallecular pouch residue was present in $69 \%$ with L, 73\% with SL, and 87\% with SS. The mean number of words recognized at phone call was 56 of 75 (range, 27-74). IOPI showed a mean tongue strength of $19.2 \mathrm{kPa}$ (range, 0-40), and a mean endurance of 16.2 seconds (range, 0-60).

Conclusion: CTS does not significantly affect speech. Sub-clinical food aspiration and vallecular pouch are present in a significant proportion of patients, especially when adjuvant treatments are administered. Residual tongue strength is not affected when proper reconstruction is performed.

Key Words: Oral cavity, microvascular reconstruction and transplant surgery, swallowing/dysphagia, speech language pathology.

Level of Evidence: 4
\end{abstract}

Laryngoscope, 00:1-8, 2020

\section{INTRODUCTION}

Treatment strategies for oral tongue/floor-of-mouth squamous cell carcinoma (OTFOMSCC) have not substantially changed in the last decades and consist, for advanced lesions, in surgery followed by adjuvant radiotherapy (RT) or chemoradiotherapy (CRT). Conventional oral surgery with macroscopic healthy margins of 1-2 cm has not been universally accepted as a proper technique to optimize local control and may present issues in terms of consistency and reproducibility. Moreover, based on

From the Department of Otorhinolaryngology - Head and Neck Surgery (A.G., N.M., F.D.B., A.P., D.L., M.F., A.D., D.L., P.N.), University of Brescia, Brescia, Italy; Department of Otorhinolaryngology (c.P.), Maxillofacial and Thyroid Surgery, Fondazione IRCCS, National Cancer Institute of Milan, Milan, Italy; Department of Oncology and Oncohaematology (c.P.), University of Milan, Milan, Italy; Department of Radiology (B.F.), University of Brescia, Brescia, Italy; and the Department of Physical Medicine and Rehabilitation (м.M.), University of Brescia, Brescia, Italy.

Editor's Note: This Manuscript was accepted for publication on February 28, 2020.

The authors declare that this manuscript was conceived and written by the cited authors, they do not have to disclose financial information, and they confirm no conflict of interest concerning this manuscript.

Send correspondence to Alberto Grammatica, MD, Department of Otorhinolaryngology-Head and Neck Surgery, University of Brescia, Piazza Spedali Civili 1, 25123 Brescia, Italy. E-mail: albertogrammatica@libero.it

DOI: 10.1002/lary.28627 anatomical studies and previous reports focusing on the diffusion pattern of OTFOMSCC, the importance of extrinsic muscles and paramedian/lateral lingual septa involvement as potential avenues for loco-regional tumor spread has been recognized to play a pivotal role when planning surgical treatment. ${ }^{1-3}$

Last update of the TNM staging system introduced the parameter of depth of infiltration (DOI) as a relevant prognosticator that is able per se to accordingly stratify different tumors. ${ }^{4,5}$ In particular, OTFOMSCC with a DOI $>10 \mathrm{~mm}$ have been demonstrated to be associated with a high risk of residual disease when treated by conventional transoral approaches. ${ }^{6-8}$ Therefore, since the seminal papers of Calabrese et al., ${ }^{9-12}$ compartmental tongue surgery (CTS) has been proposed in advanced lesions with the intent to remove the tumor en bloc, within the entire hemitongue and floor-of-mouth compartment, along with the T-N tract and draining lymph nodes, thus standardizing the surgical technique and improving loco-regional control. The authors compared long-term oncological outcomes of CTS with those of a historical cohort of patients treated by standard transoral surgery and found an improvement in 5-year local, locoregional controls, and overall survival of $16.8 \%, 24.4 \%$, and $27.3 \%$, respectively. ${ }^{10}$ Similar favorable results were 
independently confirmed by our group, at least when considering naive tumors, but not in the salvage setting. ${ }^{13}$

One of the main concerns regarding CTS is represented by a presumed excessive impairment of speech and swallowing, possibly derived from the removal of the entire hemitongue and floor-of-mouth compartment, with ensuing flap reconstruction. The aim of this study was therefore to investigate the long-term functional outcomes in patients treated by CTS and reconstructed by free flaps for advanced stage OTFOMSCC by objective and subjective evaluations of swallowing and speech.

\section{MATERIALS AND METHODS}

This study was conducted in a tertiary academic referral hospital (Department of Otorhinolaryngology-Head and Neck Surgery, University of Brescia, Italy) from March 2008 to October 2017 on 48 patients consecutively treated with CTS followed by fasciocutaneous free flap reconstruction for naive OTFOMSCC with a DOI > $10 \mathrm{~mm}$ (Table I). Inclusion and exclusion criteria are summarized in Table II. Four patients were excluded from the study for death occurring during the study period: two for loco-regional relapse, one for distant metastases, and one for other non-oncological disease.

Preoperative work-up encompassed tumor biopsy, contrastenhanced magnetic resonance (MR) for DOI evaluation, neck ultrasonography (US), and positron emission tomography (PET). All patients received tracheotomy and nasogastric feeding tube (NGFT). Antibiotic prophylaxis (ampicillin sulbactam +/- metronidazole) was routinely administered.

The CTS technique has been described in detail in previous studies. ${ }^{3,9-11,13}$ Reconstruction was accomplished in all patients by either radial forearm (RF) or anterolateral thigh (ALT) free flaps.

Postoperative swallowing and speech rehabilitation under the guidance of a dedicated speech therapist started at bedside in the immediate postoperative course (seventh postoperative day or later if intervening complications).

A few patients $(\mathrm{n}=10 \%, 20.8 \%)$ were evaluated at 6 months from the end of treatment (including adjuvant therapy, when indicated). On the other hand, all patients received functional assessment after 12 months. Patients were submitted to objective tests as

\section{TABLE I.}

Demographic and Oncologic Data.

\begin{tabular}{lc}
\hline No. of Patients & 48 \\
\hline Mean age, yr (range) & $59(26-86)$ \\
No. of patients over-70 & $13(27 \%)$ \\
M:F ratio & $2: 1$ \\
ASA score 2 & $31(64.6 \%)$ \\
ASA score 3 & $17(35.4 \%)$ \\
Pull-through approach & $44(92 \%)$ \\
Transmandibular approach & $4(8 \%)$ \\
Marginal mandibulectomy & $2(4 \%)$ \\
Unilateral neck dissection & $33(69 \%)$ \\
Bilateral neck dissection & $15(31 \%)$ \\
Type of free flap & RF 32 (67\%)ALT 16 (33\%) \\
Oropharyngeal involvement & $14(30 \%)$ ALT (9/14; 64.3\%)RF (5/14; 35.7\%) \\
Adjuvant (C)RT & $33(69 \%) C R T ~ 13(27 \%) R T ~ 20(42 \%)$
\end{tabular}

ASA = American Society of Anesthesiologists; ALT = antero-lateral thigh; $(\mathrm{C}) \mathrm{RT}=$ (chemo)radiotherapy; $\mathrm{RF}$ = radial forearm
TABLE II.

Inclusion and Exclusion Criteria for CTS.

\begin{tabular}{lc}
\hline Inclusion Criteria & Exclusion Criteria \\
\hline SCC of oral tongue/floor of mouth & $\begin{array}{c}\text { MR finding of massive transgression } \\
\text { of the median lingual raphe with } \\
\text { bilateral tongue compartments } \\
\text { involvement }\end{array}$ \\
Malor involvement of other oral \\
subsites, oropharynx, and/or \\
medullary infiltration of the \\
mandible
\end{tabular}

Postoperative survival $>1$ year

$\mathrm{ALT}=$ antero-lateral thigh; $(\mathrm{C}) \mathrm{RT}=$ (chemo)radiotherapy; $\mathrm{DOI}=$ depth of infiltration; $\mathrm{MR}=$ magnetic resonance; $\mathrm{RF}=$ radial forearm; $\mathrm{SCC}=$ squamous cell carcinoma.

videonasal endoscopic evaluation (VEES) and videofluoroscopy (VFS) for swallowing assessment, phone call for evaluation of speech articulation, and tongue strength and endurance testing (Fig. 1A,B).

VEES was performed with colored semiliquid bolus and coupled with transoral evaluation of oral/oropharyngeal postswallowing pooling. VFS was carried out by oral administration of liquid (L), semiliquid (SL), and semisolid (SS) contrast medium (barium) in the right lateral and antero-posterior projections with digital registration of swallowing (30 frames/sec) using a Siregraph CF (Siemens, Forchheim, Germany).

All VEES and VFS examinations were recorded and graded according to a 3-point scale proposed by Donzelli et al. ${ }^{14}$ : level 1 , no laryngeal vestibule food entering; level 2 , laryngeal vestibule food entering without penetration or aspiration; level 3, tracheal aspiration. Other parameters evaluated were delayed swallowing and oral/pharyngeal post-swallowing pooling. VEES were blindly and separately estimated by three independent otolaryngologists. The same otolaryngologists and one dedicated radiologist graded each VFS examination in the same way.

We selected particular combinations of tongue shapes, places, and manners of speech articulation to test the most resection-influenced phonemes (Fig. 2). During a phone call, patients were asked to read a list of 75 words containing these phonemes to an inexpert listener. Subsequently, a score ranging from 0 to 5 points (according to the number of words recognized for each single phoneme) was assigned (Table III).

The Iowa Oral Performance Instrument (IOPI) produced by the IOPI Medical (Woodinville, WA, USA) is a validated diagnostic and therapeutic device that objectively assesses tongue elevation strength by measuring the maximum pressure (expressed in $\mathrm{kPa}$ ) on a standard sized air-filled bulb by pressing it between the hard palate and tongue (Fig. 1A,B). ${ }^{15}$ It allows for comparing patient data to that in normal subjects (50th percentile of tongue strength, $60.7 \mathrm{kPa}){ }^{16}$ IOPI can be used to assess tongue fatiguability by measuring its endurance (inversely proportional to the former). Endurance is measured by quantifying the time (in seconds) that a patient can maintain $50 \%$ of the maximum pressure. In this setting, we used the IOPI as a measure of postoperative function: for each patient, we performed three tests, with 60 -second pauses. The final results for strength and endurance were the mean values of the three tests.

Subjective tests for function evaluation were also administered in all patients after 1 year from the end of treatment. Specifically, the EORTC H\&N35 (questions 35, 36, 37, and 38) and 


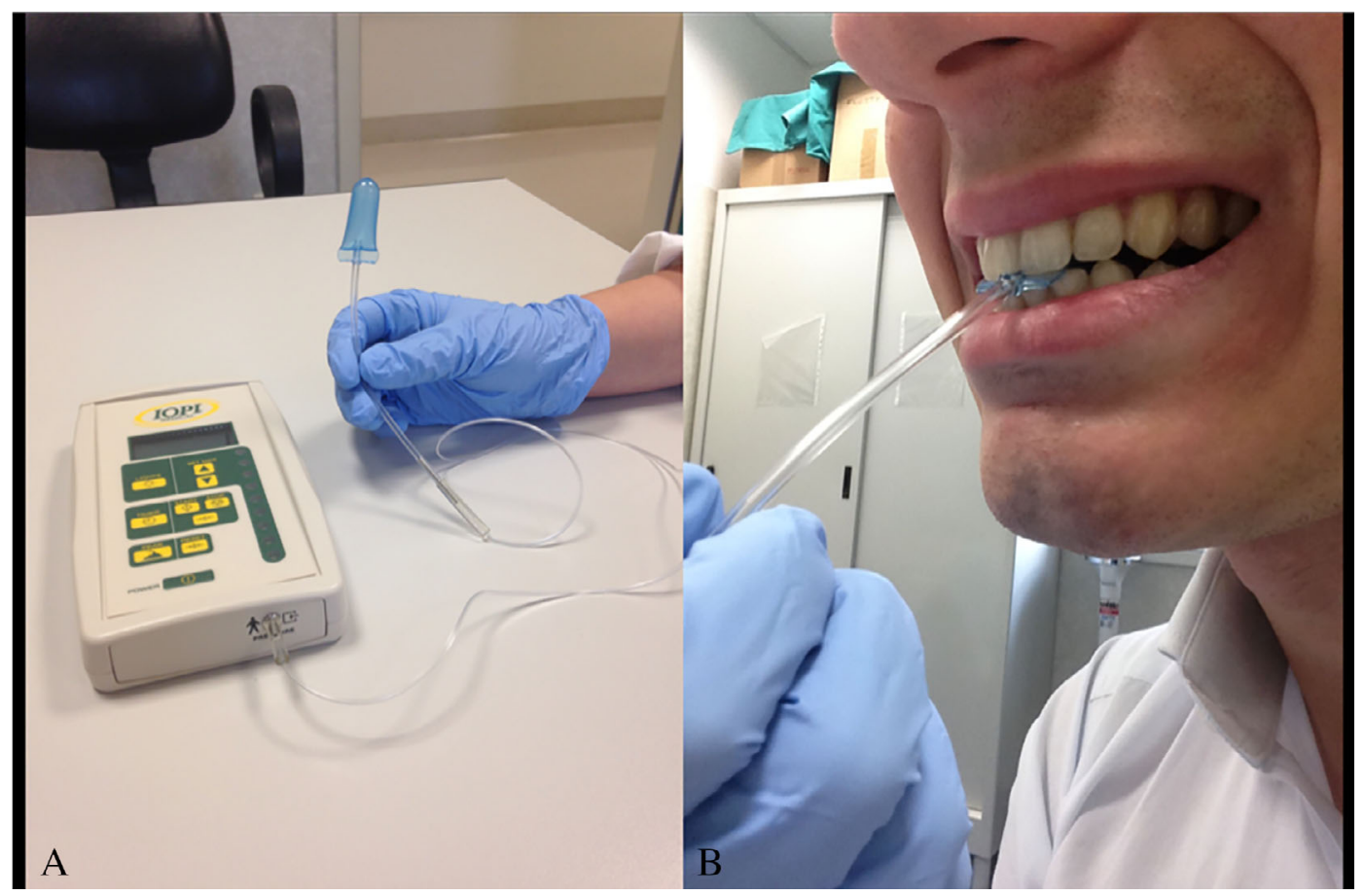

Fig. 1. (A) lowa Oral Performance Instrument (IOPI) device. (B) intraoral position of IOPI during patient evaluation. [Color figure can be viewed in the online issue, which is available at www.laryngoscope.com.]

UWQOL v.4 (question 5) questionnaires were adopted for swallowing. EORTC H\&N35 (questions 53, and 54) and UWQOL v.4 (question 7) were used for evaluation of speech function.

\section{Statistical Analysis}

Statistical analysis was performed with JMP Pro14 software (SAS Institute Inc., Cary, North Carolina, USA). Parameters that significantly influenced the objective functional outcomes were evaluated with logistic regression analysis to

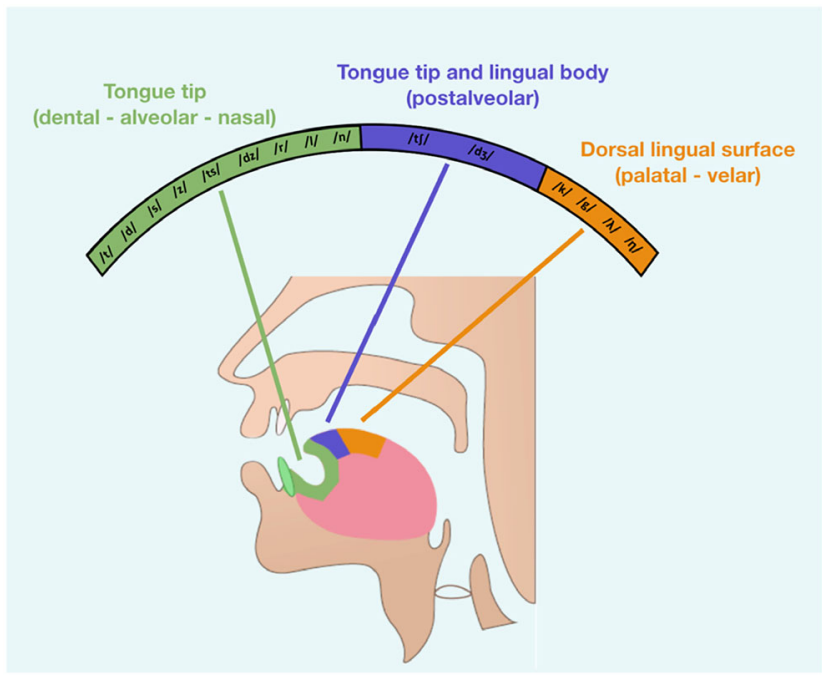

Fig. 2. Schematic drawing of phonemes tested for places and manners of tongue articulation after surgery. [Color figure can be viewed in the online issue, which is available at www.laryngoscope.com.] simultaneously adjust for different confounders. The technique requires a dichotomous dependent $(\mathrm{Y})$ and a set of independent variables $(\mathrm{X})$ that are classified as prognostic factors. Independent variables were: evaluation time (6 vs. 12 months), age (more or less than 70 years), death occurred before or after 1 year from treatment (yes vs. no), type of flap used (RF vs. ALT), resection of oropharyngeal structures (yes vs. no), and adjuvant treatments (yes vs. no). Dichotomous dependent variables of the analyzed logistics model were: VEES, VFS, telephone call, resistance, and pressure tests. For the apex, body and posterior tongue speech articulation scores, as well as for pressure and resistance tests, the corresponding averages were taken as cut-off values.

With the logistic regression and the related odds ratio, we evaluated the risk (or probability) of the response of each $\mathrm{Y}$ according to the above-mentioned $\mathrm{X}$ variables that were supposed to be correlated with the course of the disease. The significance of the logistic model was evaluated with the chi-square (Whole Model) and the Wald tests for validity of the effects.

\section{RESULTS}

All patients were postoperatively decannulated (mean tracheotomy dependence time, 10 days), and $2(4 \%)$ remained gastrostomy-tube dependent at 1 year from the end of treatment.

Concerning microsurgical reconstructive outcomes, no total free flap failure nor donor site surgical complication occurred. Recipient site minor complications were observed in two patients: one cervical seroma and one pharyngocutaneous fistula, both healed with conservative treatments. Major complications (requiring return to the operatory room) were observed in four cases: one need for redo-venous anastomosis, one revision for hemorrhage, one $\mathrm{RF}$ free flap partial necrosis salvaged by a pectorals 
TABLE III.

Phonemes Tested for Speech Articulation.

\begin{tabular}{|c|c|c|c|c|c|}
\hline & & \multicolumn{3}{|c|}{ Combinations of Tongue Shapes and Places of Articulation } & \multirow[b]{2}{*}{ Total Score } \\
\hline & & Tongue Tip & Tongue Tip and Lingual Body & Dorsal Lingual Surface & \\
\hline \multirow[t]{3}{*}{ Manners } & Stop & $/ \mathrm{t} / \mathrm{d} /$ (dental) & & $/ \mathrm{k} / \mathrm{g} /$ (velar) & \\
\hline & Affricative & /ts/ /dz/ (dental) & $/ \mathrm{t} \int / / \mathrm{d} 3 /$ (postalveolar) & & \\
\hline & Approximant & $/ r /$ (alveolar) & & & \\
\hline Scores & & $0-45$ & $0-10$ & $0-20$ & $0-75$ \\
\hline
\end{tabular}

major pedicled flap, and one drainage of oro-cervical fistula with neck abscess due to flap dehiscence.

\section{Early Evaluation (6 Months)}

VEES. The rate of aspiration classified according to the Donzelli scale was level 1 in $60 \%(\mathrm{n}=6)$, level 2 in $30 \%(\mathrm{n}=3)$, and level 3 in $10 \%(\mathrm{n}=1)$. Post-deglutition residue was present at the level of the vallecular pouch in eight $(80 \%)$ patients, glosso-tonsillar sulcus in seven (70\%), piriform sinus in five $(50 \%)$, and floor of mouth in three $(30 \%)$. Prolonged swallowing time was observed in six $(60 \%)$ patients.

VFS. The percentage of patients showing Donzelli level 1,2 , or 3 were $10 \%(\mathrm{n}=1), 20 \%(\mathrm{n}=2)$, and $70 \%$ $(\mathrm{n}=7)$ with $\mathrm{L} ; 40 \%(\mathrm{n}=4), 30 \%(\mathrm{n}=3)$, and $30 \%(\mathrm{n}=3)$ with SL; and 50\% ( $\mathrm{n}=5), 30 \%(\mathrm{n}=3)$, and 20\% $(\mathrm{n}=2)$ with SS, respectively. Vallecular pouch residue rate was $80 \%(\mathrm{n}=8)$ with $\mathrm{L}, 50 \%(\mathrm{n}=5)$ with $\mathrm{SL}$, and $100 \%$ ( $\mathrm{n}=10$ ) with SS. We observed piriform sinus residue in $30 \%(\mathrm{n}=3), 30 \%(\mathrm{n}=3)$, and $50 \%(\mathrm{n}=5)$ of cases with $\mathrm{L}$, SL, and SS, respectively. Finally, we observed a prolonged swallowing time with L, SL, and SS in 30\% ( $\mathrm{n}=3), 40 \%$ $(\mathrm{n}=4)$, and $70 \%(\mathrm{n}=7)$ of patients, respectively.

Speech evaluation. The mean number of words correctly recognized by an inexpert listener during a phone call with the patient were 52 (range, 32-68). The number and distribution of recognized words according to the overall phonemic type are detailed in Figure 2.

Objective evaluation of tongue strength and endurance. Mean strength was $15.4 \mathrm{kPa}$ (range, 3-32), while mean endurance was 15 seconds (range, 0-60).

\section{Late Evaluation (12 Months)}

VEES. The percentages of aspiration classified according to the Donzelli scale was: level 1 in $67 \%$ $(\mathrm{n}=32)$, level 2 in $23 \%(\mathrm{n}=11)$, and level 3 in $10 \%$ $(\mathrm{n}=5)$. Post-deglutition residue was present at the level of the vallecular pouch in 39 patients (81\%), glossotonsillar sulcus in $32(67 \%)$, piriform sinus in $19(40 \%)$, and floor-of-mouth in $16(33 \%)$. Prolonged swallowing time was observed in 16 patients (33\%).

VFS. The percentages of patients with Donzelli level 1,2 , or 3 were $42 \%(n=20), 25 \%(n=12)$, and $33 \%(n=16)$ with $\mathrm{L} ; 48 \%(\mathrm{n}=23), 19 \%(\mathrm{n}=9)$, and $33 \%(\mathrm{n}=16)$ with SL; and 54\% ( $\mathrm{n}=25), 33 \%(\mathrm{n}=15)$, and $13 \%(\mathrm{n}=6)$ with SS, respectively. Two patients did not undergo VFS with SS due to aspiration and discomfort in the L and SL steps of VFS. Vallecular pouch residue was present in $69 \%(\mathrm{n}=33)$ with L, $73 \%(\mathrm{n}=35)$ with SL, and $87 \%(\mathrm{n}=40)$ with SS. We detected piriform sinus residue in 50\% ( $\mathrm{n}=24), 40 \%$ $(\mathrm{n}=19)$, and $37 \%(\mathrm{n}=17)$ of cases with $\mathrm{L}, \mathrm{SL}$, and SS, respectively. Finally, we observed a prolonged swallowing time with $\mathrm{L}$, SL, and SS in $8 \%(\mathrm{n}=4), 17 \%(\mathrm{n}=8)$, and $37 \%$ ( $\mathrm{n}=15$ ) of patients, respectively.

Speech evaluation. The mean number of words correctly recognized was 56 (range, 22-74).

Objective evaluation of tongue strength and endurance. IOPI device evaluation was performed in 44 patients (92\%). Mean strength was $19.2 \mathrm{kPa}$ (range, 0-40), mean endurance was 16.2 seconds (range, 0-60).

Subjective test evaluation at 12 months. For swallowing function, the mean score of EORTC H\&N35 was: $<60 \%$ in $16.7 \%(\mathrm{n}=8), 60 \%$ to $80 \%$ in $18.7 \%(\mathrm{n}=9)$, and $>80 \%$ in $64.6 \%(\mathrm{n}=31)$. The UWQOL v.4 was: $<60 \%$ in $22.9 \%(\mathrm{n}=11), 60 \%$ to $80 \%$ in $58.3 \%(\mathrm{n}=28)$, and $>80 \%$ in $18.8 \%(\mathrm{n}=9)$.

For speech articulation, the mean score of EORTC H\&N35 was: $<60 \%$ in $16.7 \%(n=8), 60 \%$ to $80 \%$ in $47.9 \%$ $(\mathrm{n}=23)$, and $>80 \%$ in $35.4 \%(\mathrm{n}=17)$. The UWQOL v.4 was: $<60 \%$ in $6.3 \%(\mathrm{n}=3), 60 \%-80 \%$ in $75 \%(\mathrm{n}=36)$, and $>80 \%$ in $18.7 \%(\mathrm{n}=9)$.

\section{Factors Influencing Functional Outcomes}

Considering VEES, vallecular pouch residue varied according to the type of postoperative treatment performed $(P=.017)$ : non-irradiated patients, those treated by $\mathrm{RT}$, and those receiving $\mathrm{CRT}$ had a $60 \%, 95 \%$, and $84.6 \%$ rate of vallecular pouch, respectively. Floor-ofmouth pooling was significantly associated with $\mathrm{RF}$ reconstruction $(40.6 \%$ vs. $18.8 \%, P=.049)$ and had a correlation with associated oropharyngeal resection $(42.9 \%$ vs. $29.4 \%, P=.073)$. Similarly, patients over 70 years of age $(50 \%$ vs. $34.3 \%, P=.064)$ had a higher rate of postdeglutition residue at the level of the piriform sinus, but the difference did not reach statistical significance.

Concerning VFS evaluations, correlations between all analyzed variables are summarized in Table IV: gender, 
TABLE IV.

Types of Food Tested at VFS and Affected Variables $(P<.05$ are evidenced in bold character, while $P<.1$ are underlined).

Liquids After $1 \mathrm{yr}$

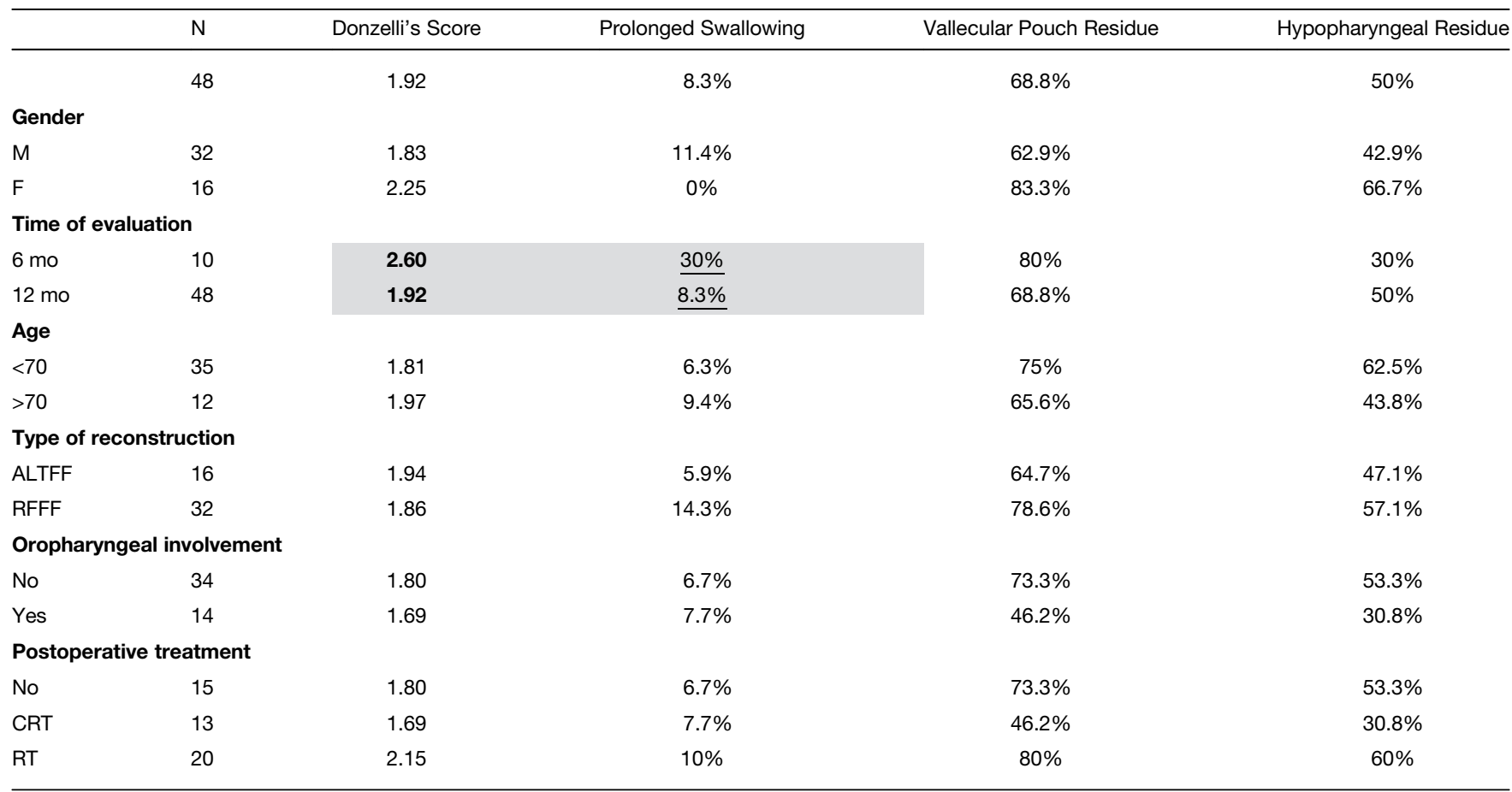

Semi-Liquids After $1 \mathrm{yr}$

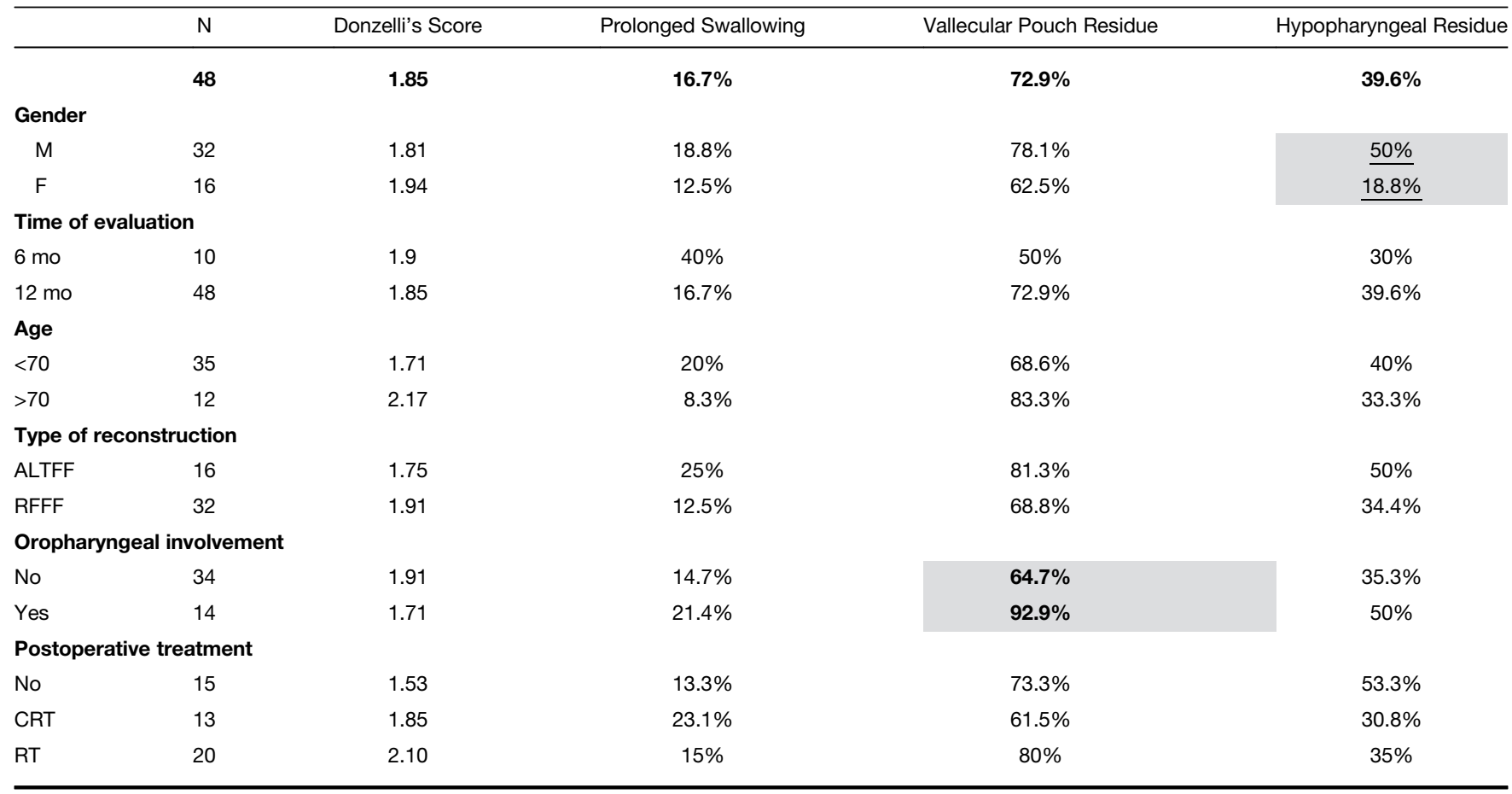

Semi-Solids After $1 \mathrm{yr}$

\begin{tabular}{lccccc}
\hline & N & Donzelli's Score & Prolonged Swallowing & Vallecular Pouch Residue & Hypopharyngeal Residue \\
\hline Gender & $\mathbf{4 8}$ & $\mathbf{1 . 5 9}$ & $\mathbf{3 2 . 6 \%}$ & $\mathbf{8 7 \%}$ & $\mathbf{3 7 \%}$ \\
M & 32 & 1.56 & $\underline{34.4 \%}$ & $\mathbf{8 7 . 5 \%}$ & $\mathbf{5 3 . 1 \%}$ \\
F & 16 & 1.64 & $\underline{28.6 \%}$ & $85.7 \%$ & $\mathbf{0} \%$ \\
\hline
\end{tabular}




\begin{tabular}{|c|c|c|c|c|c|}
\hline \multicolumn{6}{|c|}{$\begin{array}{l}\text { TABLE IV. } \\
\text { Continued }\end{array}$} \\
\hline \multicolumn{6}{|c|}{ Semi-Solids After $1 \mathrm{yr}$} \\
\hline & $\mathrm{N}$ & Donzelli's Score & Prolonged Swallowing & Vallecular Pouch Residue & Hypopharyngeal Residue \\
\hline \multicolumn{6}{|c|}{ Time of evaluation } \\
\hline $6 \mathrm{mo}$ & 10 & 1.70 & $70 \%$ & $100 \%$ & $50 \%$ \\
\hline $12 \mathrm{mo}$ & 48 & 1.59 & $32.6 \%$ & $87 \%$ & $37 \%$ \\
\hline \multicolumn{6}{|l|}{ Age } \\
\hline$<70$ & 35 & 1.52 & $30.3 \%$ & $81.8 \%$ & $33.3 \%$ \\
\hline$>70$ & 12 & 1.75 & $41.7 \%$ & $100 \%$ & $41.7 \%$ \\
\hline \multicolumn{6}{|c|}{ Type of reconstruction } \\
\hline ALTFF & 16 & 1.56 & $37.5 \%$ & $93.8 \%$ & $62.5 \%$ \\
\hline RFFF & 32 & 1.60 & $30 \%$ & $83.3 \%$ & $23.3 \%$ \\
\hline \multicolumn{6}{|c|}{ Oropharyngeal involvement } \\
\hline No & 34 & 1.64 & $33.3 \%$ & $84.8 \%$ & $33.3 \%$ \\
\hline Yes & 14 & 1.46 & $30.8 \%$ & $92.3 \%$ & $46.2 \%$ \\
\hline \multicolumn{6}{|c|}{ Postoperative treatment } \\
\hline No & 15 & 1.36 & $7.1 \%$ & $85.7 \%$ & $50 \%$ \\
\hline CRT & 13 & 1.46 & $38.5 \%$ & $76.9 \%$ & $30.8 \%$ \\
\hline RT & 20 & 1.84 & $47.4 \%$ & $94.7 \%$ & $31.6 \%$ \\
\hline
\end{tabular}

postoperative adjuvant treatment, oropharyngeal extension, and time of evaluation ( 6 vs. 12 months) were the most important variables influencing functional outcomes.

Patients receiving postoperative treatment had a tendency toward delayed swallowing times with SS (nonirradiated $7.1 \%$, CRT $38.5 \%$, RT $47.4 \%, P=.006$ ). Oropharyngeal extension had a higher rate of vallecular pouch residue with SL $(92.9 \%$ vs. $64.7 \%, P=.045)$ and higher Donzelli scores with SS (1.64 vs. 1.46, $P=.087$ ). Furthermore, patients evaluated 6 months after surgery achieved higher Donzelli scores with L $(P=.023)$, and more prolonged swallowing time with $\mathrm{SS}(P=.038)$.

There was also a significant correlation between speech outcomes in terms of words recognized at phone and flap choice, with better results observed using $\mathrm{RF}$ (59.25 vs. $49.63, P=.020)$.

Males (19.87 kPa vs. $17.62 \mathrm{kPa}, P=.048)$ and patients submitted to $\mathrm{RF}$ reconstruction $(20.07 \mathrm{kPa}$ vs. $17.69 \mathrm{kPa}$, $P=.005$ ) had better results at objective strength evaluation. Considering endurance, better results were associated with age over 70 years (19.68 vs. 6.82 seconds, $P=.028$ ).

Patients excluded from the study $(n=4)$ for death occurrence after less than 1 year from the end of treatment, underwent evaluation at 6 months in three cases only. The outcomes in this subset did not show statistically significant differences when compared to the remnant of the study cohort. Multivariate analysis did not evidence death in the first year after surgical treatment as a negative factor impacting functional outcomes of CTS.

\section{DISCUSSION}

Since the first description of CTS for OTFOMSCC, different authors have confirmed its good oncologic outcomes, whereas precise data regarding functional results are still lacking. For the first time in a comprehensive and quantitative way, our study precisely evaluated objective and subjective functional outcomes of patients undergoing CTS and reconstructed by fasciocutaneous free flaps.

Our data showed that patients in the present series experienced a non-negligible incidence of sub-clinical aspiration although at VEES evaluation none was classified as level 3 according to the Donzelli scale, and VFS aspiration rates ranged from $33 \%$ to $13 \%$ (according to the type of food). Residues at the level of the vallecular pouch and hypopharynx were frequent with L, SL, and SS food consistencies. Finally, a minority of patients showed a prolonged swallowing time with L (8\%) and SL (17\%). However, $37 \%$ of subjects showed a prolonged swallowing time with SS. Speech intelligibility was optimal, with a mean of 56 of 75 words recognized during a phone call. Furthermore, most patients had satisfying to near-normal results, with only a minority of subjects showing poor rates of intelligibility, thus favorably comparing with the results in the literature. ${ }^{17,18}$ In particular, Chang et al. ${ }^{17}$ defined word intelligibility of $>80 \%$ as score 4 (out of 4), finding similar speech quality results after hemiglossectomies, with optimal results in most of patients (mean score, 3.74).

Of note, tongue strength and endurance were objectively evaluated using the IOPI device: at our assessment after 1 year, RF reconstruction seems to guarantee better results in terms of residual muscular strength and can be explained in terms of better residual tongue movements when not tethered by a bulky flap.

Overall, the most important variables influencing deglutition were adjuvant treatment, age, gender, oropharyngeal resection, and type of free flap employed, as previously reported in literature. ${ }^{19-23}$ Performing functional evaluation at 6 and 12 months showed that it is reasonable to expect some improvements up to 1 year after 
surgery, as also confirmed by other studies. ${ }^{24-27}$ It is interesting to note how at 1 year after treatment patients' subjective perception of swallowing is generally reported as good/satisfactory in more than $50 \%$ of cases using both the EORTC and UWQOL questionnaires. On the other hand, speech function is less favorable and considered moderate in more than $50 \%$ of subjects, especially when evaluated by the UWQOL questionnaire.

Our series is characterized by a standardized and homogeneous surgical approach requiring flap reconstruction to prevent salivary fistula or tethering of the residual tongue. Thus, from a functional standpoint, resection and reconstruction can be considered as a rather uniform baseline for these patients, and the other variables (age, CRT, oropharyngeal resection, and type of flap) can be evaluated in each individual patient. Calabrese et al. ${ }^{10,11}$ and Piazza et al. ${ }^{13}$ previously identified the oncological indications of CTS for OTFOMSCC in detail. This study may further help in correctly defining the patient population best suited for this surgical approach, contributing to preoperative counseling and reduction of undesired side effects. Speech intelligibility is often optimal regardless of patient characteristics, while swallowing may be partially impaired in elderly patients and those receiving postoperative (C)RT. Furthermore, the relevant degree of subclinical aspiration observed should advise some caution in patients with compromised pulmonary function and guide their dietary modifications to reduce the amount of adverse events. ${ }^{28,29}$

The role of reconstruction after tongue surgery is still debated. As a general rule, the functional impact of complex reconstructive techniques should always be weighed against the increased risk of complications. ${ }^{30}$ More extensive defects generally lead to poorer speech and swallowing results as demonstrated by Hartl et al., ${ }^{31}$ with the volume of resection considered as a major predictor of swallowing and aspiration-related issues when evaluated by the EORTC H\&N35 questionnaire. This underlines the significant impact of the resection size, suggesting that reconstruction is primarily adopted to guarantee adequate oncological margins. Moreover, Ji et al. ${ }^{32}$ recently demonstrated a significant difference between partial glossectomies and hemiglossectomies, showing that reconstruction led to functional impairments in the former. When not strictly needed, flaps may add excessive bulk to the residual tongue by reducing its range of movements; on the other hand, while potentially impairing speech, such adjunctive bulk may help in deglutition. ${ }^{33,34}$

Therefore, it is essential to find the adequate balance between the type of defect and the type of flap selected for each kind of reconstruction. In fact, adequate donor site selection according to each individual defect has the potential to result in consistent functional outcomes regardless of the type of flap employed. ${ }^{35,36}$ On this basis, a CTS approach represents the ideal setting due to the relatively standardized size, shape of defect, and amount of neural (lingual and hypoglossal nerves) and muscular (intrinsic and extrinsic muscles) structures routinely sacrificed according to the present surgical technique. Furthermore, most authors report significant deterioration of speech and swallowing only in patients requiring resections wider than an hemiglossectomy, thus involving the contralateral tongue compartment or the mandible. ${ }^{17,21,23}$ In this view, when appropriately indicated, CTS for OTFOMSCC has the potential to offer superior oncologic results without significantly reducing functional outcomes compared to conventional partial glossectomy.

\section{CONCLUSIONS}

CTS for oral cancer demonstrated good survival outcomes especially as a primary treatment modality, whereas no data on functional results have been gathered up to now. Our study demonstrated that this type of surgery does not substantially affect speech function. The main issue remains the swallowing process, causing some $\mathrm{L}$ and SS food aspiration and vallecular food pouch residue, especially when postoperative treatments are administered. Residual tongue strength does not seem to be affected when proper reconstruction with $\mathrm{RF}$ is accomplished. Some functional improvements were both subjectively as well as objectively observed up to 1 year after the end of treatment in most of patients.

\section{BIBLIOGRAPHY}

1. Boland PW, Pataridis K, Eley KA, Golding SJ, Watt-Smith SR. A detailed anatomical assessment of the lateral tongue extrinsic musculature, and proximity to the tongue mucosal surface. Does this confirm the current TNM T4a muscular subclassification? Surg Radiol Anat 2013;35:559-564.

2. Sanders I, Mu L. A three-dimensional atlas of human tongue muscles. Anat Rec (Hoboken) 2013;296:1102-1114.

3. Piazza C, Montalto N, Paderno A, Taglietti V, Nicolai P. Is it time to incorporate "depth of infiltration" in the T staging of oral tongue and floor of mouth cancer? Curr Opin Otolaryngol Head Neck Surg 2014;22:81-89.

4. Brierley JD, Gospodarowicz MK, Wittekind C. TNM Classification of Malignant Tumours. 8th ed. Union for International Cancer Control. Oxford: Wiley Blackwell; 2017.

5. Amin MB, Edge S, Greene F, et al. AJCC Cancer Staging Manual. 8th ed. New York: Springer; 2017.

6. Soo KC, Carter RL, O'Brien CJ, Barr L, Bliss JM, Shaw HJ. Prognostic implications of perineural spread in squamous carcinomas of the head and neck. Laryngoscope 1986;96:1145-1148.

7. Steinhart H, Kleinsasser O. Growth and spread of squamous cell carcinoma of the floor of the mouth. Eur Arch Otorhinolaryngol 1993;250:358-361.

8. Ganly I, Goldstein D, Carlson DL, et al. Long-term regional control and survival in patients with "low-risk," early stage oral tongue cancer managed by partial glossectomy and neck dissection without postoperative radiation: the importance of tumor thickness. Cancer 2013;119:1168-1176.

9. Calabrese L, Giugliano G, Bruschini R, et al. Compartmental surgery in tongue tumours: description of a new surgical technique. Acta Otorhinolaryngol Ital 2009;29:259-264.

10. Calabrese L, Bruschini R, Giugliano G, et al. Compartmental tongue surgery: long term oncologic results in the treatment of tongue cancer. Oral Oncol 2011;47:174-179.

11. Calabrese L, Tagliabue M, Maffini F, Massaro MA, Santoro L. From wide excision to a compartmental approach in tongue tumors. Curr Opin Otolaryngol Head Neck Surg 2013;21:112-117.

12. Tagliabue $\mathrm{M}$, Gandini $\mathrm{S}$, Maffini $\mathrm{F}$, et al. The role of the T-N tract in advanced stage tongue cancer. Head Neck 2019;41:2756

13. Piazza C, Grammatica A, Montalto N, Paderno A, Del Bon F, Nicolai P. Compartmental surgery for oral tongue and floor of the mouth cancer: oncologic outcomes. Head Neck 2019;41:110-115.

14. Donzelli J, Brady S, Wesling M, Craney M. Predictive value of accumulated oropharyngeal secretions for aspiration during video nasal endoscopic evaluation of the swallow. Ann Otol Rhinol Laryngol 2003;112:469-475.

15. VanRavenhorst-Bell HA, Mefferd AS, Coufal KL, Scudder R, Patterson J. Tongue strength and endurance: comparison in active and non-active young and older adults. Int $J$ Speech Lang Pathol 2017;19:77-86.

16. IOPI MEDICAL. Normal values. Available at: https://iopimedical.com/ normal-values/. Accessed September 12, 2019

17. Chang EI, Yu P, Skoracki RJ, Liu J, Hanasono MM. Comprehensive analysis of functional outcomes and survival after microvascular reconstruction of glossectomy defects. Ann Surg Oncol 2015;22:3061-3069.

18. Furia CL, Kowalski LP, Latorre MR, et al. Speech intelligibility after glossectomy and speech rehabilitation. Arch Otolaryngol Head Neck Surg 2001;127:877-883. 
19. Li X, Sun Q, Guo S. Functional assessments in patients undergoing radial forearm flap following hemiglossectomy. J Craniofac Surg 2016;27: e172-e175.

20. Fujiki M, Miyamoto S, Zenda S, Sakuraba M. Longitudinal and long-term effects of radiotherapy on swallowing function after tongue reconstruction. $J$ Laryngol Otol 2016;130:865-872.

21. Ohkoshi A, Ogawa T, Nakanome A, et al. Predictors of chewing and swallowing disorders after surgery for locally advanced oral cancer with free flap reconstruction: a prospective, observational study. Surg Oncol 2018;27:490-494.

22. Shin YS, Koh YW, Kim S-H, et al. Radiotherapy deteriorates postoperative functional outcome after partial glossectomy with free flap reconstruction. J Oral Maxillofac Surg 2012;70:216-220.

23. Suzuki H, Hyodo I, Hasegawa Y. Prediction of decannulation, oral intake recovery, overall survival and lung metastasis following oral malignant tumor resection and reconstruction. Oncol Lett 2018;15:2686-2694.

24. Canis M, Weiss BG, Ihler F, Hummers-Pradier E, Matthias C, Wolff HA. Quality of life in patients after resection of pT3 lateral tongue carcinoma: microvascular reconstruction versus primary closure. Head Neck 2016;38 89-94.

25. Yuan Y, Zhang $\mathrm{P}, \mathrm{He} \mathrm{W}, \mathrm{Li}$ W. Comparison of oral function: free anterolateral thigh perforator flaps versus vascularized free forearm flap for reconstruction in patients undergoing glossectomy. J Oral Maxillofac Surg 2016;74:1500.e1-e6.

26. Villaret AB, Cappiello J, Piazza C, Pedruzzi B, Nicolai P. Quality of life in patients treated for cancer of the oral cavity requiring reconstruction: a prospective study. Acta Otorhinolaryngol Ital 2008;28:120-125.

27. Lam L, Samman N. Speech and swallowing following tongue cancer surgery and free flap reconstruction - A systematic review. Oral Oncol 2013;49: 507-524.
28. Daggett A, Logemann J, Rademaker A, Pauloski B. Laryngeal penetration during deglutition in normal subjects of various ages. Dysphagia 2006;21: 270-274.

29. Brown L, Rieger JM, Harris J, Seikaly H. A longitudinal study of functional outcomes after surgical resection and microvascular reconstruction for oral cancer: tongue mobility and swallowing function. $J$ Oral Maxillofac Surg 2010;68:2690-2700.

30. Khan MN, Perez E, Goljo E, et al. The price of free tissue transfer after tongue reconstruction: quantifying the risks. Laryngoscope 2017;127: 1551-1557.

31. Hartl DM, Dauchy S, Escande C, Bretagne E, Janot F, Kolb F. Quality of life after free-flap tongue reconstruction. J Laryngol Otol 2009;123: $550-554$.

32. Ji YB, Cho YH, Song CM, et al. Long-term functional outcomes after resection of tongue cancer: determining the optimal reconstruction method. Eur Arch Otorhinolaryngol 2017;274:3751-3756.

33. Hsiao H-T, Leu Y-S, Lin C-C. Tongue reconstruction with free radial forearm flap after hemiglossectomy: a functional assessment. J Reconstr Microsurg 2003;19:137-142.

34. Tarsitano A, Vietti MV, Cipriani R, Marchetti C. Functional results of microvascular reconstruction after hemiglossectomy: free anterolateral thigh flap versus free forearm flap. Acta Otorhinolaryngol Ital 2013;33: 374-379.

35. de Vicente JC, de Villalaín L, Torre A, Peña I. Microvascular free tissue transfer for tongue reconstruction after hemiglossectomy: a functional assessment of radial forearm versus anterolateral thigh flap. J Oral Maxillofac Surg 2008;66:2270-2275.

36. Lu M, Sun G, Hu Q, Tang E, Wang Y. Functional assessment: free thin anterolateral thigh flap versus free radial forearm reconstruction for hemiglossectomy defects. Med Oral Patol Oral Cir Bucal 2015;20:e757-e762. 